Chapter 17

\title{
New Approaches to the Psychological Treatment of Obsessive-Compulsive Disorder in Adults
}

\author{
Clare Rees and Rebecca Anderson \\ Additional information is available at the end of the chapter \\ http://dx.doi.org/10.5772/53070
}

\section{Introduction}

The key features of Obsessive-Compulsive Disorder (OCD) are the experience of recurrent, unwanted and intrusive thoughts (obsessions) and/or the completion of repetitive ritualistic behaviours (compulsions). Compulsions are frequently performed to reduce the distress associated with the obsessional thoughts [1]. The lifetime prevalence of OCD is estimated to be $2-3 \%$ in the general population, with epidemiological studies indicating consistency in rates across different countries and cultures $[2,3]$.

OCD is a severe anxiety disorder that is associated with significant disability. In fact, the World Health Organisation has rated OCD as the tenth leading cause of disability in the world. If left untreated, OCD typically becomes a chronic problem with a pattern of frequent re-occurrence and relapse [2]. Unfortunately, many individuals will significantly delay helpseeking, due to reasons such as a fear of embarrassment, and thus suffer for years with significant symptoms and distress [4,5].

OCD is typically diagnosed according to Diagnostic and Statistical Manual of Mental Disorders criteria [1]. These stipulate that the individual must experience either obsessions, compulsions, or both, and that these must interfere with the individual's life due to the time they consume (i.e., more than one hour per day) or by causing marked distress or significant impairment. To obtain an accurate diagnosis, clinicians will often use structured diagnostic interviews such as the Mini International Neuropsychiatric Interview (MINI) [6], Anxiety Disorders Interview Schedule (ADIS-IV) [7], or Structured Clinical Interview for the Diagnostic and Statistical Manual of Mental Disorders (SCID) [8]. Furthermore, clinicians and researchers will frequently utilize a selection of measures to ascertain symptom severity and to assess for clinical outcomes. The most common of these include the Yale-Brown Obses- 
sive Compulsive Symptom Checklist (YBOCS) [9], and the Obsessive Compulsive Inventory - Revised (OCI-R) [10].

\section{Why new approaches to treatment are needed}

For some time now, the dominant approach to treating this disorder is a behavioural therapy called Exposure and Response Prevention (ERP). ERP is considered the gold-standard treatment as it currently has the most convincing evidence of efficacy from several controlled trials [11] and metaanalytic studies [12] spanning a number of years. In a study examining the change scores as a result of treatment outcomes, Fisher and Wells [13] found that 50-60\% of participants were likely to be classified as recovered following ERP treatment. ERP is typically delivered on an individual basis and may be implemented as a stand alone treatment, or combined with cognitive strategies under the label of Cognitive Behaviour Therapy (CBT). Despite the success of ERP in alleviating the symptoms of OCD for many individuals, there remains much scope for further improving the success of psychological treatments for the disorder. Issues such as treatment refusal, non-completion of ERP, non-response in some cases and restricted accessibility are just some problems effecting outcomes.

Presently, there are major issues with costs and limited psychological therapy resources in the public healthcare system [14]. There are very limited therapeutic services available to the public in the area of OCD, and the majority of individuals do not have access to good treatments. According to Shafran and colleagues [15], evidence suggests that even though a number of evidence-based psychological treatments have been developed, clients are not receiving them within clinical settings. Furthermore, when evidence-based psychological treatments are delivered, they are often not delivered to an optimal standard [15]. Shafran and colleagues [15] provided a number of recommendations to approach this gap in welldelivered evidence-based psychological treatments, however, it may take time to increase awareness and knowledge about evidence-based psychological treatments, as well as the implementation of them. Given the lack of available therapeutic services being provided to the public as well as the delivery of evidence-based psychological treatments, a stepped care approach could provide another solution in the meantime to this problem [14].

A stepped care approach simply refers to treatments at differing intensities [14]. Within a stepped care approach, the treatment must be the least restrictive, but still provide significant improvements to health. With this in mind, if a treatment is provided at a lower level or step, and the client is not improving post-treatment, the client's needs should be evaluated, and an appropriate step to treatment provided. The restrictiveness of a treatment generally refers to the cost and personal inconvenience. When referring to the therapist, it can also refer to the availability of the therapists time and the amount of time required by a specialist therapist [14]. Given the limited number of therapists trained in the area of OCD, a stepped care approach appears to be particularly warranted in the treatment of OCD.

The National Institute for Health and Clinical Excellence (NICE) Guidelines for Obsessivecompulsive disorder [16], a world-renowned document outlining evidence-based ap- 
proaches to treating the disorder, endorses the use of a stepped-care model in the prevention and provision of treatment services to individuals with OCD. The NICE Guidelines outline six levels of intervention as follows. It should be noted that all references to CBT within this stepped care model emphasize the need to include an ERP component in therapy.

Step 1: Awareness and recognition

Step 2: Recognition and assessment (including referral to appropriate services)

Step 3: Initial treatment by guided self-help and CBT via group or individual format

Step 4: For OCD with comorbidity or a poor response to initial treatment - treatment via CBT within a multidisciplinary team, possible medication

Step 5: For OCD with significant comorbidity, severe impairment or limited treatment response - specialist treatment services with expertise in CBT, possible medication

Step 6: Inpatient or intensive treatment programs including medication and CBT to reduce the risk to life, severe self-neglect or severe distress or disability.

As can be seen, while the higher level steps within this care model indicate the need for ERP based psychotherapy, which is provided on an individual basis, there is much scope for exploring less intensive, less costly, and more widely accessible formats for the delivery of ERP based treatment programs.

Furthermore, it is important to review emerging and alternate approaches to the conceptualization and treatment of this disorder. The capacity for ERP based treatments to bring about considerable and clinically significant change for many clients is well documented. However, prior studies have noted that up to $25 \%$ of patients refuse ERP treatment due to the time commitments needed to complete the treatment, a fear that the exposure exercises will bring about overwhelming anxiety, or a fear that a dreaded outcome will occur should the individual fail to complete their rituals [17]. Within this stepped care model there are limited evidence-based alternatives for treatment other than medication-based treatment, which individuals may also refuse or be unable to tolerate. It is therefore important that we continue to explore new psychological treatment approaches for this disorder and thus offer hope to those who refuse or do not respond to the current gold-standard treatment approaches.

\section{New Treatment Approaches}

The following section will provide a review of a number of new approaches to the treatment of OCD. First, the use of treatment modalities that differ from standard individual based CBT or ERP will be discussed. This will explore the use of the group therapy format, and the use of modern technologies in the delivery of cognitive and behavioural therapies. Next, emerging conceptualizations and treatment approaches will be discussed. This will review the evidence for Metacognitive and Acceptance and Commitment based approaches to the management of OCD. Each approach will first be described, the proposed mechanisms of change outlined and then a critical review of the evidence for efficacy will be offered. 


\section{Group therapy for OCD}

The predominant groups that have been evaluated for OCD are either cognitive, behavioural or a combination of both. Provision of therapy in groups for OCD makes intuitive sense because of the known issues relating to shame, isolation and embarrassment that have been identified amongst sufferers. Being able to meet others with a similar set of problems can allow for symptoms to be normalized and destigmatised. Participating in a group can allow the individual to develop a support network for sharing strategies, information and resources, and the process of helping others may enhance ones self-esteem, and sense of social competence and connectedness. From a clinical perspective, peer modeling of ERP exercises may provide additional opportunities for exposure and reinforce compliance with homework. In addition to these potential advantages, as already mentioned, stepped-care models emphasize the importance of providing treatment options which are less costly and intensive. Group therapy formats have the capacity to increase access to well-trained clinicians, and decrease potential waiting times.

\section{Empirical Evidence for Group CBT for OCD}

A meta-analysis by Jónsson and Hougaard [18] examined the effectiveness of group CBT and ERP as reported in thirteen prior treatment trials. This included randomized controlled trials, non-randomized controlled trials, and naturalistic trials. The meta-analysis indicated that group CBT and ERP yields a large overall pre-post effect size of 1.18. Importantly, group dropout rates of $13.5 \%$ were compared with reported individual treatment dropout rates of $12.1 \%$. This was considered an indication that the group format seemed an acceptable method of treatment delivery to most patients. When this information is combined with the earlier findings that direct comparisons between group and individual based treatments have not demonstrated a significant difference in post treatment outcomes [19,20], group CBT and ERP certainly appear to be viable and efficacious treatment options in the management on this disorder. Furthermore, the meta-analysis indicated that compared to pharmacological treatment, the between groups effect size of 0.80 favoured group CBT/ERP.

Since this meta-analysis, Belotto-Silva and colleagues [21] compared group CBT (GCBT) with Fluoxetine, a Selective Serotonin Reuptake Inhibitor, in a controlled trial including 158 participants (GCBT N=70; Fluoxetine $\mathrm{N}=88$ ). A total of 12 group therapy sessions of 2 hours duration were provided each week. The Fluoxetine group received $80 \mathrm{mg} /$ day for the same duration (12 weeks). Mean YBOCS scores decreased by 23\% (GCBT) and 21\% (Fluoxetine). The authors note that this level of symptom reduction, although significant, is less than has been observed in some other CBT studies. They conclude that this is most likely due to the high rate of comorbidity present in the sample. In fact the results are closer to other similar studies that have utilised less stringent exclusion criteria and therefore arguably more representative samples [19,22]. In another study since the meta-analysis [23], the effectiveness of GCBT for OCD was further evaluated in a representative, clinic sample in Norway. In this open trial, 54 patients diagnosed with OCD were provided with 12 sessions of GCBT. At post treatment and again at 3 and 12 month follow-up significant improvements in OCD symptoms as well as depression and anxiety were observed. 
It could be argued that these outcomes are simply due to the non-specific effects of participating in a group, such as the increased social support that participants experience. A study by Fineberg and colleagues [24] therefore attempted to account for the role of non-specific group effects within their research design by including a relaxation placebo control group. The outcomes indicated that both those in the CBT and relaxation placebo group improved, and that there was no significant difference between the outcomes for the two groups. However, there was a significant bias towards non-uptake of the relaxation therapy condition. With more potential participants dropping out prior to the trial even beginning, this may mean that the final relaxation group participants represented individuals predisposed to believe that relaxation was a credible form of treatment for OCD and that they therefore had enhanced expectations of treatment. There was also a significant difference in drop-out rates across conditions with $35 \%$ of participants dropping out of the relaxation group compared to $4 \%$ in the CBT group. Due to the observed difficulties with finding a credible group placebo, we are not yet able to ascertain whether these results are simply due to the non-specific group factors. However, this study yielded a large treatment effect size of 1.45 for GCBT, providing further support for the further use of group CBT.

Overall, the large effect sizes noted in prior studies demonstrate that group CBT and ERP are viable treatment options for service provision, with comparable findings to individual therapy and pharmacological treatments for OCD. Although further group placebo controlled trials may go some way to separating out the effects of non-specific group factors, the consistent reports of clinically and statistically significant improvements for participants, and the potential improved access to expert services for clients and cost savings for clinicians indicate that group therapy is a worthy option in the treatment of this disorder.

\section{Telemental Health Approaches}

Telemental health is a term used to capture the broad application of telecommunication and information technology in the provision of various mental health services. There are a number of different applications that have been developed and trialed for the treatment of OCD in adults. A summary of the evidence base and key advantages and disadvantages of these applications is provided in Table 1.

$\begin{array}{lll}\text { Evidence } & \text { Decrease Improves access to Im Other potential } & \text { Other potential } \\ \text { sphysical expert clinicians } & \text { pa advantages } & \text { disadvantages } \\ \text { barriers } & \text { ct } & \\ \text { to } & \text { on } & \text { ti } \\ \text { treatmen } & \text { m } \\ \text { t } & \text { e } \\ & \text { re } \\ & \text { qu } \\ & \text { ire } \\ \text { d }\end{array}$




\begin{tabular}{|c|c|c|c|c|}
\hline & & & & $\begin{array}{l}\text { of } \\
\text { cli } \\
\text { ni } \\
\text { cia } \\
\mathrm{n}\end{array}$ \\
\hline \multicolumn{5}{|c|}{ Telephone-based } \\
\hline $\begin{array}{l}\text { No therapist } \\
\text { (automated) }\end{array}$ & RCTs & Yes & No & $\begin{array}{ll}\text { Eli Able to be completed at } & \text { Isolation, lack of } \\
\text { mi time selected by client } & \text { therapeutic alliance may } \\
\text { na } & \text { impact on treatment } \\
\text { te } & \text { adherence and } \\
\text { s } & \text { retention }\end{array}$ \\
\hline With therapist & RCTs & Yes & Yes & $\begin{array}{l}\text { De Regular contact may Lack of non-verbal cues } \\
\text { cr improve treatment } \\
\text { ea compliance, support for } \\
\text { se clients experiencing } \\
\text { s difficulty with program }\end{array}$ \\
\hline \multicolumn{2}{|c|}{ Video-conferencing Case series } & Yes & Yes & $\begin{array}{ll}\text { N Able to be conducted on } & \text { Observation of non- } \\
\text { o mobile devices and } & \text { verbal cues may be } \\
\text { ch home computers, } & \text { limited by screen set-up, } \\
\begin{array}{cl}\text { an presence of non-verbal } & \text { clients may not be } \\
\text { ge cues, observation of } & \text { familiar or comfortable } \\
\text { exposure exercises } & \text { with technology }\end{array}\end{array}$ \\
\hline \multicolumn{5}{|l|}{ Internet-based } \\
\hline No therapist & Open trial & Yes & No & $\begin{array}{ll}\text { Eli Able to be completed at Isolation, lack of } \\
\text { mi time selected by client } & \text { therapeutic alliance may } \\
\text { na } & \text { impact on treatment } \\
\text { te } & \text { adherence and retention } \\
\text { s } & \end{array}$ \\
\hline With therapist & Open trial & Yes & Yes & $\begin{array}{l}\text { De Regular contact may Lack of non-verbal cues } \\
\text { cr improve treatment } \\
\text { ea compliance, support for } \\
\text { se clients experiencing } \\
\text { s difficulty with program }\end{array}$ \\
\hline Virtual Reality & Case series & Yes & No & $\begin{array}{ll}\text { De Technology may enable } & \text { Virtual environment } \\
\text { cr treatment to be } & \text { may not extend to cater } \\
\text { ea conducted on mobile } & \text { for idiosyncratic } \\
\text { se devices and home } & \text { presentations } \\
\text { s computers } & \end{array}$ \\
\hline
\end{tabular}

Table 1. Advantages and disadvantages of telemental health approaches 


\section{Telephone-Based Treatments}

BT-Steps [25] is a well-evaluated behaviour therapy self-help program that is administered via the telephone. It does not involve direct therapist contact as clients simply access an automated system where they enter details of their progress and receive automatic suggestions and prompts from an electronic recording. Reductions in YBOCS symptoms of up to $30 \%$ have been reported in open studies of patients who completed the self-exposure phase of BT-Steps [26] however a major limitation of this approach is the unusually high dropout rate that has been observed in the studies. In attempt to investigate this further, Kenwright, Marks, Graham, Franses and Mataix-Cols [27] compared the self-administered BT-Steps to a BT-Steps with inclusion of nine sessions of therapist support. Those in the supported condition evidenced both improved compliance and outcomes.

Three trials of CBT via telephone with therapist contact have been conducted with increasingly improved methodological rigor. Lovell and colleagues [28] first conducted a case series with four participants examining the impact of eight weekly telephone contacts with a cognitive behavioural therapist, starting and ending with a face-to-face session. Three out of the four participants improved, with reductions of between $24 \%$ to $67 \%$ noted on the YBOCS. In a later trial, Taylor and colleagues [29] provided 12 weekly therapy sessions by phone and with provision of a self-help book and compared this to a waitlist control. Thirty three participants were included and results indicated significant reduction in symptoms for the telephone group. More recently, Lovell and colleagues [30] compared telephone-based treatment with face-to-face CBT in a non-inferiority trial involving 72 participants. In each condition participants received 10 therapy sessions however, the telephone sessions were half the duration of the face-to-face sessions. Equivalent improvements were found across both groups suggesting that the telephone condition may represent an effective treatment with considerable reduction in therapist's time requirements.

\section{Videoconferencing}

Videoconferencing is a computer-assisted approach that bears the closest resemblance to face-to-face therapy because both therapist and client interact in real-time. It has major advantages in terms of eliminating the issue of distance between therapist and client. It is particularly beneficial for clients who have restricted mobility for any reason or who might not otherwise be inclined to leave their homes to attend therapy sessions. To date, two studies have explored the treatment of OCD via videoconference. Himle and colleagues [31] utilised a multiple-base line design to analyse the outcomes of a 12-week manualised CBT intervention. Three participants were included in the study and significant improvements in symptoms were observed for all participants as measured by the YBOCS. Participants also reported high satisfaction with the therapy and excellent scores on therapeutic alliance were also obtained.

In a recent case series design, Vogel and colleagues [32] investigated the effectiveness of videoconferencing therapy with the addition of cell phones. Fifteen sessions of ERP was delivered via six teleconference and nine cell phone sessions. Four of the six participants made substantial gains and no longer met criteria for OCD at the end of therapy. Importantly, 
these gains were maintained at a three month follow-up, and all patients rated the treatment format as acceptable and that the working alliance with the therapist was high. The authors did note that these results should be interpreted with caution due to the small sample size, lack of a control comparison and blind ratings, and possible selection bias of participants where those who are more comfortable with technologies self-select into the study. However, it was also recognized that the addition of cell phones to the trial allowed for a back-up when teleconferencing equipment did not work, and allowed the clinician to monitor exposure exercises conducted away from the videoconferencing equipment. With the increasing number of cell phones allowing video calls, the potential for this portable technology to improve the capacity for in-situ exposure exercises should only improve with time.

\section{Internet-Based CBT}

Treatments that are delivered via computers enable a client to gain assistance from remote locations or from the convenience of their own homes. For some time now, less costly and intense forms of intervention such as bibliotherapy and self-directed exposure have been recognized as an effective option for provision of psychological treatment [33]. More recently, bibliotherapy has been brought into the technological age with the development of computerized versions that as per their original counterparts, do not include any therapist contact. However, on the whole, the evidence has suggested that better results are achieved with the addition of minimal therapist support [34].

A recent open trial of an 8-week CBT treatment ('The OCD Program') delivered via the Internet has been completed [35]. Twenty-two individuals with a primary diagnosis of OCD participated in the trial receiving eight online CBT lessons in addition to twice-weekly telephone contact from a Clinical Psychologist. It should be noted that the average amount of therapist contact time was only 86 minutes across the 8 weeks. Significant improvements in YBOCS total scores were found with a reported within-groups effect size of 1.28 at posttreatment. This suggests that significant improvements in symptoms may be possible with only very limited therapist input.

In another recent open trial [36], 23 patients diagnosed with OCD were provided with a 15week Internet-Based CBT program with therapist support. Consistent with the Wootton study, therapist contact time was minimal (average of 92 minutes per patient across the entire 15 weeks) and the within-groups effect size on the YBOCS was large (1.56). Taken together, the results of these trials suggest that CBT provided over the Internet with only minimal therapist time can result in substantial symptom reduction of a similar magnitude to that seen in face-to-face studies. However, as these are only open trials randomized controlled trials with larger samples and comparisons against face-to-face CBT are required to strengthen the conclusions.

\section{Virtual Reality}

Riva and Gamberini [37] assert that "virtual reality is an application that lets users navigate and interact with a three-dimensional computer-generated environment in real time" (p. 327). Virtual reality can be conducted on a variety of different systems ranging from 3-dimension software installed on a personal computer to immersive headsets connected to a 
hand control [38]. Virtual reality therapies are based on the assumption that people feel "present" in the virtual environment, whereby at some level their perception fails to recognize the role of technology in their experience [39]. A particular advantage of virtual reality systems is that they have the potential to offer accurate standardization and replication of a prescribed environment [40].

Virtual reality therapies have been demonstrated to be more effective than no treatment for several specific phobias, including spider phobia, acrophobia, and a fear of flying [39]. However, head-to-head trials with gold-standard in-vivo ERP treatments are limited, and the value of this form of treatment over the current leading approach is therefore unclear at this stage. The potential cost effectiveness of exposure via a virtual reality system is most obvious for problems where stimuli for the exposure exercise may be difficult or expensive to come by (e.g., flights for exposure exercises to address a fear of flying, or spiders to address a spider phobia). Virtual reality therapies therefore remain a promising area for future directions in anxiety disorder treatment, but require further evaluation at this stage.

In the only study evaluating virtual reality for OCD to date, Clark, Kirkby, Daniels, and Marks [41] used an interactive virtual environment to provide vicarious exposure to dirt for thirteen individuals with OCD. Participants completed three 45-minute computer based treatment sessions at weekly intervals. During the session, the participants were instructed to direct a figure on the screen to engage in exposure, such as by touching dirt in a virtual garden, and encouraged to refrain from selecting that the figure wash their hands in the virtual sink. Participants were awarded points for exposing their figure to dirt, and then for refraining from washing their hands despite the figure rating their anxiety as high. Following treatment, participants showed a significant decrease on their depression scores and on one measure of OCD symptoms, the Padua Inventory. However, no significant change was noted on their YBOCS scores.

In a follow-up report on the same study, it was noted that across the three virtual reality sessions participants became faster at engaging in hand dirtying, and were less likely to engage in hand-washing in the virtual environment [42], indicating that participants were able to learn the principles of ERP via this format. Given the low dose of therapy applied in this trial (i.e., three sessions only), and capacity to provide this treatment without ongoing therapist input, this therapy format certainly warrants further investigation to ascertain whether learning from the virtual environment can generalize to real-world experiences. The main limitation to future development of virtual reality programs for OCD may be the need to create a sufficient range of virtual environments and activities to cater for the expansive variety of OCD symptom presentations beyond contamination fears and associated hand-washing.

\section{Summary of Empirical Evidence for Telemental Health Treatment of OCD}

Taken together, the field of telemental health treatment for OCD is very promising with a number of controlled trials now showing that treatments administered via computer with minimal or no therapist assistance can result in significant reductions in obsessive-compulsive symptoms. Particularly promising results are being seen in Internet-Based treatments 
whereby as little as ten minutes of telephone contact per week from a therapist is associated with large effect sizes on obsessive-compulsive symptoms. While some approaches may enable reductions in therapist contact hours, approaches such as video-conferencing may not reduce contact hours but instead enable individuals with OCD to receive expert treatment regardless of geographical location. It is therefore envisaged that future treatments within a stepped care model could utilize computer and internet based therapies at a lower step, with technologies such as video-conferencing being used to provide expertise for individuals with high levels of comorbidity or who have not responded to less intense treatment formats who would otherwise have difficulty accessing such services. While the use of virtual reality based programs requires further investigation, it is possible that should it be demonstrated as an effective treatment format, it could be built into current computer or internet based self-help programs as a stand-alone treatment, or as a component in an overall treatment which also includes psychoeducation and the development of a personalized ERP hierarchy.

\section{Metacognitive Therapy}

Metacognition refers to beliefs about thinking and strategies used to regulate and control thinking and was originally elaborated upon by Flavell [43]. Since then a number of theorists have incorporated aspects of metacognition into various psychological models of OCD [44-47]. Most notable amongst these theorists is Adrian Wells who, along with colleagues, has developed a comprehensive metacognitive account of OCD based on the Self Regulatory Executive (S-REF) model [48,49]. According to this model, a style of thinking called the cognitive attentional syndrome (CAS) is the main causal factor in prolonging all emotional disorders. However, Wells has gone on to specify which particular aspects of this model are most relevant to understanding OCD. Wells and Mathews [48] and Wells [46] propose that three types of metacognitive knowledge are important in the etiology and maintenance of symptoms: thought fusion beliefs, beliefs about the need to perform rituals, and criteria that signal rituals can be stopped. In this model, thought fusion beliefs are extended beyond thought action fusion (belief that having a thought increases the chance of acting on it) but also thought-event fusion (the belief that having a thought can cause an event or means that an event has happened) and thought object fusion (the belief that thoughts or feelings can be transferred into objects). According to the model the three overall types of metacognitive knowledge operate in a causal chain to explain obsessive-compulsive symptoms.

The main approach used in MCT for OCD is to help the client to become aware of their metacognitive processing and to learn to modify these higher order metacognitions such as beliefs about the importance of thoughts. MCT differs from standard CBT in that no attempts are made to modify lower order appraisals such as 'I am responsible to ensure nothing happens to my family'. Also, MCT does not include exposure exercises aimed at habituation but instead includes the use of behavioural experiments to assist in the interruption of unhelpful metacognitive processes such as attempts to suppress thoughts.

Empirical Evidence for MCT for OCD 
Two small trials of MCT for adults with OCD have been conducted to date. The first case series trial included four individuals diagnosed with OCD who received 12 sessions of individual MCT [50]. Substantial reduction in obsessive-compulsive symptoms was found with all participants meeting clinical significance criteria for recovery at post-treatment and 3month follow-up. A second open trial included 8 individuals diagnosed with OCD who received 12 sessions of group-based MCT [51]. Similar results were found in this study with seven out of eight participants achieving recovery according to the YBOCS clinical significance criteria. This result was maintained at 3-month follow-up.

Steffen Moritz and colleagues [52] developed and tested a self-help treatment called 'My Metacognitive Training for OCD' (myMCT) which contains a mixture of standard cognitivebehavioural elements plus the addition of metacognitive treatment elements. They recruited 86 individuals with OCD over the Internet and randomly assigned them to either the selfhelp or wait-list condition. Those in the myMCT condition were emailed an electronic version of the self-help book. Participants who received the intervention showed significantly greater reductions in obsessive-compulsive symptoms compared to the wait-list group. It should be noted that this treatment did contain traditional cognitive restructuring elements and is thus less of a 'pure' test of metacognitive treatment for OCD.

Given that all bar one participant in the earlier MCT trials achieved recovery status and had maintained this at 3-month follow-up, MCT may represent a promising way forward for clinicians and patients alike. As this therapeutic approach does not directly rely on exposure exercises, it may provide a less aversive form of therapy for those who in the past have refused to start or have initiated but then dropped out of ERP programs. The small samples sizes, lack of control conditions, and possible non-specific effects of participating in a group program do limit the conclusions of these trials. Furthermore, there has been no comparison between MCT and gold-standard ERP to date. Further research could go some way towards determining whether MCT fits within the stepped care model of treatment as an efficacious stand alone individual based treatment, an alternative to group ERP or CBT, or as a self-help based program.

\section{Acceptance and Commitment Therapy}

ACT is based on a philosophy of functional contextualism and the Relational Frame Theory (RFT) [53]. The basic premise of this theory is that individuals learn relationships between stimuli and responses through a number of different processes and this relationship is not necessarily contingent upon actual experience with that stimuli. For example, in the case of OCD a person may fear contamination from handrails because they are similar or grouped together with shopping trolleys which are considered 'dirty'. ACT consists of six main therapy processes that are not targeted in a linear fashion but rather are addressed within therapy when the opportunity or need arises. The six therapy processes are

1. Acceptance,

2. Defusion,

3. Self as context, 
4. Contact with the present moment,

5. Values, and

6. Committed Action [54].

Unlike conventional CBT, ACT does not concern itself with attempts to address the content of cognitions or behaviours in OCD but rather the processes. For example, in ACT the client with OCD is assisted to see how previous attempts to control obsessions have failed and that the focus of therapy will shift to 'accept' rather than 'struggle' to eradicate obsessional thoughts. The therapist realigns the client to consider improvement in quality of life as the goal rather than reducing or eliminating symptoms. Another major aspect of the therapy is to help the clients achieve 'cognitive defusion', in other words, to see thoughts less literally. Instead of attempting to analyse, rationally challenge or evaluate the accuracy of thoughts, clients are assisted to 'defuse' from obsessions and to alter their relationship to these experiences by treating them just as thoughts in one's head or 'relatively unimportant words' [55]. Various therapeutic exercises are used to assist the client to achieve defusion such as 'thanking' the mind for a thought. 'Self as context' simply refers to the process of helping the client to separate his or her inner experiences from self. For example, to understand that obsessional thoughts are separate from who the client is as a person. Mindfulness exercises are also used to help the client to become more aware of current experiences, such as thoughts and sensations and not to engage in avoidance but to simply observe and pay attention to those experiences. Values work in ACT focuses on helping the client to follow through on actions or behaviours that are more consistent with personally held values (e.g., being a good friend). This is in contrast to behaviours that are aimed at avoiding anxiety (e.g., completing cleaning rituals). Finally, behavioural commitment exercises are designed to ensure that the client continues to engage in behaviours that are consistent with their values. For example, making time once a week to visit friends would be a behavioural commitment.

Although ACT is described as a Behaviour Therapy and it does include exposure exercises, the difference is that ACT does not utilise these therapeutic strategies with the aim of reducing rituals or distress per se. Rather, all of the strategies are used to help the client practice acceptance and mindfulness whilst heading in valued directions. Often there will be a simultaneous reduction in frequency and distress of obsessions and compulsions as a function of other more functional behaviours taking precedence but this is not the focus of the therapy [55].

\section{Empirical Evidence for ACT with OCD}

Twohig, Hayes, and Masuda [56] conducted a multiple baseline study of ACT for OCD that included four clients with different symptom presentations (two checking, one cleaning, one hoarding). The intervention consisted of 8 hours of ACT. At the end of treatment, significant reductions in symptoms were obtained on the Obsessive Compulsive Inventory (OCI) with results maintained at 3-month follow-up. This same ACT protocol was later compared to a progressive relaxation training (PRT) control condition in the first randomized controlled trial of ACT for OCD [57]. The study included 79 adults diagnosed with OCD; with primary symptoms measured using the YBOCS. Significant improvements on the YBOCS were found for the ACT condition at post treatment and follow-up. In addition, clinically signifi- 
cant improvement occurred more in the ACT compared to the PRT condition. Although this study provides initial empirical support for the efficacy of ACT for OCD, further controlled trials are required as well as trials including longer follow-up periods and comparisons against other active and well established treatments such as ERP.

\section{Summary and Conclusion}

While ERP has long been established as the most effective treatment for OCD, the typical application of the treatment in an individual face-to-face format may limit client access to effective therapy and specialist clinicians. The stepped-care model suggests that lower intensity treatment formats, such as self-help and group therapy, be utilized so that only those with more complex needs are referred to the higher intensity individual format. This review has indicated that internet based CBT programs with minimal therapist support and group ERP based therapy are supported as lower intensity treatment options within a stepped care model. There is limited evidence that CBT conducted over the telephone or via videoconferencing will reduce therapist time working with patients. These formats therefore do not offer an alternative step within the stepped care model, but instead offer an additional means for delivering the higher intensity step of individual based therapy which is less susceptible to geographical or physical barriers to attending therapy. At this stage, further evaluations are required of virtual reality based programs to ascertain their effectiveness either as a stand-alone treatment or as a component within a treatment package.

Although still considered the gold-standard treatment, some patients will decline to start or complete ERP based therapy, and a proportion will not improve despite application of the treatment by expert clinicians. It is noted that the stepped care model offers limited evidence-based psychological alternatives to ERP based psychotherapy. It is therefore important that we continue to evaluate alternative approaches. Early indications from MCT and ACT trials have shown promising results. However, for both approaches the studies have involved small samples, and neither treatment has been directly compared with ERP in a clinical trial. Until such a comparison takes place, no conclusion can be made as to whether these approaches should be included as an option within the current stepped care model.

\section{Author details}

Clare Rees* and Rebecca Anderson

*Address all correspondence to: c.rees@curtin.edu.au

School of Psychology and Speech Pathology, Curtin Health Innovation Research Institute (CHIRI), Curtin University, Australia 


\section{References}

[1] American Psychiatric Association. (2000). Diagnostic and statistical manual of mental disorders. Text revision ( $4^{\text {th }}$ ed.). Washington: Author.

[2] Abramowitz, J., Taylor, S., \& Mc Kay, D. (2009). Obsessive-Compulsive Disorder. The Lancet, 374(9688), 491-499.

[3] Pallanti, S. (2008). Transcultural observations of obsessive-compulsive disorder. The American Journal of Psychiatry, 165, 169-170.

[4] Rasmussen, S., \& Tsuang, M. (1986). Clinical characteristics and family history in DSM-III obsessive-compulsive disorder. American Journal of Psychiatry, 143, 317-322.

[5] Steketee, G. (1993). Treatment of obsessive-compulsive disorder. New York: Guilford Press.

[6] Sheehan, D., Lecrubier, Y., Sheehan, K., Amorim, P., Janavs, J., Weiller, E., Hergueta, T., Baker, R., \& Dunbar, G. (1998). The Mini-International Neuropsychiatric Interview (M.I.N.I): the development and validation of a structured diagnostic psychiatric interview for DSM-IV and ICD-10. Journal of Clinical Psychiatry, 59(20), 22-33.

[7] Brown, T., Di Nardo, P., Lehman, C., \& Campbell, L. (2001). Reliability of DSM-IV anxiety and mood disorders: Implications for the classification of emotional disorders,. Journal of Abnormal Psychology, 110, 49-58.

[8] First, M., Spitzer, R., Gibbon, M., \& Williams, J. (1996). Structured Clinical Interview for DSM-IV Axis I Disorders- Patient Edition (SCID-I/P, Version 2.0). New York: Biometrics Research Department, New York State Psychiatric Institute.

[9] Goodman, W., Price, L., Rasmussen, S., Mazure, C., Fleischman, R. Hill, et al. (1989). The Yale-Brown Obsessive Compulsive Scale: Development, use, and reliability. Archives of General Psychiatry, 46, 1006-1011.

[10] Foa, E., Kozak, M., Salkovskis, P., Coles, M., \& Amir, N. (1998). The validation of a new obsessive-compulsive disorder scale: The obsessive-compulsive inventory. Psychological Assessment, 10, 206-214.

[11] Foa, E., Liebowitz, M., Kozak, M., Davies, S., Campeas, R., Franklin, M., Huppert, J., Kjernisted, K., Rowan, V., Schmidt, A., Simpson, H., \& Tu, X. (2005). Randomized, placebo-controlled trial of exposure and ritual prevention, clomipramine, and their combination in the treatment of obsessive-compulsive disorder. American Journal of Psychiatry, 162, 151-161.

[12] Kobak, K., Greist, J., Jefferson, J., Katzelnick, D., \& Henk, H. (1998). Behavioral versus pharmacological treatments of obsessive compulsive disorder: A meta-analysis. Psychopharmacology, 136, 205-216. 
[13] Fisher, P., \& Wells, A. (2005). How effective are cognitive and behavioural treatments for obsessive-compulsive disorder? A clinical significance analysis. Behaviour Research and Therapy, 43, 1543-1558.

[14] Bower, P., \& Gilbody, S. (2005). Stepped care in psychological therapies: Access, effectiveness and efficiency. British Journal of Psychiatry, 186, 11-17.

[15] Shafran, R., Clark, D., Fairburn, C., Arntz, A., Barlow, D., Ehlers, A., et al. (2009). Mind the gap: Improving the dissemination of CBT. Behaviour Research and Therapy, 47, 902-909.

[16] National Collaborating Centre for Mental Health. (2005). Obsessive compulsive disorder: Core interventions in the treatment of obsessive compulsive disorder and body dysmorphic disorder. Leicester: British Psychological Society.

[17] Greist, J. (1998). The comparative effectiveness of treatments for obsessive-compulsive disorder. Bulletin of the Meninger Clinic, 62, A65-A81.

[18] Jónsson, H., \& Hougaard, E. (2009). Group cognitive behavioural therapy for obsessive-compulsive disorder: A systematic review and meta-analysis. Acta Psychiatrica Scandinavica, 119, 98-106.

[19] Anderson, R., \& Rees, C. (2007). Group versus individual cognitive-behavioural treatment for obsessive-compulsive disorder: a controlled trial. Behaviour Research and Therapy, 45(1), 123-137.

[20] Fals-Stewart, W., Marks, A., \& Schafer, J. (1993). A comparison of behavioral group therapy and individual behavior therapy in treating obsessive-compulsive disorder. The Journal of Nervous and Mental Disease, 181, 189-193.

[21] Belotto-Silva, C., Belo, Diniz. J., Marino, Malavazzi. D., Valerio, C., Fossaluza, V., Borcato, S., Seixas, A., Morelli, D., Miguel, E., \& Shavitt, R. (2012). Group cognitivebehavioral therapy versus selective serotonin reuptakeinhibitors for obsessive-compulsive disorder: A practical clinical trial. Journal of Anxiety Disorders, 26, 25-31.

[22] Tolin, D., Maltby, N., Diefenbach, G., Hannan, S., \& Worhunsky, P. (2004). Cognitive behavioral therapy for medication non-responders with obsessive-compulsive disorder: a wait-list-controlled open trial. Journal of Clinical Psychiatry, 65(7), 922-931.

[23] Håland, A., Vogel, P., Lie, B., Launes, G., Pripp, A., \& Himle, J. (2010). Behavioural group therapy for obsessive-compulsive disorder in Norway: An open communitybased trial. Behaviour Research and Therapy, 48, 547-554.

[24] Fineberg, N., Hughes, A., Gale, T., \& Roberts, A. (2005). Group cognitive behaviour therapy in obsessive-compulsive disorder (OCD): A controlled study. International Journal of Psychiatry in Clinical Practice, 9, 257-263.

[25] Marks, I., Baer, L., Greist, J., Park, J., Bachofen, M., Nakagawa, A., Wenzel, K., Parkin, J., Manzo, P., Dottl, S., \& Mantle, J. (1998). Home self-assessment of obsessive com- 
pulsive disorder. Use of a manual and a computer-conducted telephone interview: two UK-US studies. British Journal of Psychiatry, 172, 406-412.

[26] Herbst, N., Voderholzer, U., Stelzer, N., Knaevelsrud, C., Hertenstein, E., Schelgl, S., Nissen, C., \& Kulz, A. (2012). The potential of telemental health applications for obsessive-compulsive disorder. Clinical Psychology Review, 32, 454-466.

[27] Kenwright, M., Marks, I., Graham, C., Frances, A., \& Mataix-Cols, D. (2005). Brief scheduled phone support from a clinician to enhance computer-aided self-help for obsessive-compulsive disorder: A Randomized Controlled Trial. Journal of Clinical Psychology, 61, 1499-1508.

[28] Lovell, K., Fullalove, K., Garvey, R., \& Brooker, C. (2000). Telephone treatment of obsessive-compulsive disorder. Behavioural and Cognitive Psychotherapy, 28, 87-91.

[29] Taylor, S., Thordarson, D., Spring, T., Yeh, A., Corcoran, K., Eugster, K., et al. (2003). Telephone-administered cognitive behavior therapy for obsessive compulsive disorder. Cognitive Behaviour Therapy, 32(1), 13-25.

[30] Lovell, K., Cox, D., Haddock, G., Jones, C., Raines, D., Garvey, R., et al. (2006). Telephone administered cognitive behaviour therapy for treatment of obsessive compulsive disorder: A randomised controlled non-inferiority trial. BMJ: British Medical Journal, 333, 883.

[31] Himle, J., Fischer, D., Muroff, J., Van Etten, M., Lokers, L., Abelson, J., \& Hanna, G. (2006). Videoconferencing-based cognitive-behavioral therapy for obsessive-compulsive disorder. Behaviour Research and Therapy, 44, 1821-1829.

[32] Vogel, P., Launes, G., Moen, E., Solem, S., Hansen, B., Haland, A., \& Himle, J. (2012). Videoconference- and cell phone-based cognitive behavioral therapy of obsessivecompulsive disorder: A case series. Journal of Anxiety Disorders, 26, 158-164.

[33] Tolin, D., Diefenbach, G., Maltby, N., \& Hannan, S. (2005). Stepped care for obsessive-compulsive disorder: A pilot study. Cognitive and Behavioral Practice, 12(4), 403-414.

[34] Palmqvist, B., Carlbring, P., \& Andersson, G. (2007). Internet-delivered treatments with or without therapist input: does the therapist factor have implications for efficacy and cost? Expert Review of Pharmacoeconomics \& Outcomes Research, 7, 291-297.

[35] Wootton, B., Titov, N., Dear, B., Spence, J., Andrews, G., Johnston, L., \& Solley, K. (2011). An internet administered treatment program for obsessive-compulsive disorder: A feasibility study. Journal of Anxiety Disorders, 25, 1102-1107.

[36] Andersson, E., Ljotsson, B., Hedman, E., Kaldo, V., Paxling, B., Andersson, G., Lindefors, N., \& Ruck, C. (2011). Internet-based cognitive behavior therapy for obsessive compulsive disorder: a pilot study. BMC Psychiatry, 11, 125.

[37] Riva, G., \& Gamberini, L. (2000). Virtual reality in telemedicine. Telemedicine Journal and E-Health, 6, 327-340. 
[38] Hoffman, H., Doctor, J., Patterson, D., Carrougher, G., \& Furness, T. (2000). Virtual reality as an adjunctive pain control during burn wound care in adolescent patients. Pain, 85, 305-309.

[39] Krijn, M., Emmelkamp, P., Olafsson, R., \& Biemond, R. (2004). Virtual reality exposure therapy of anxiety disorders: A review. Clinical Psychology Review, 24, 259-281.

[40] Rizzo, A., Buckwalter, J., Humphrey, L., van der Zaag, C., Bowerly, T., Chua, C., Neumann, U., Kyriakakis, C., van Rooyen, A., \& Sisemore, D. (2000). The virtual classroom: A virtual environment for the assessment and rehabilitation of attention deficits. CyberPsychology and Behavior, 3, 483-499.

[41] Clark, A., Kirkby, K., Daniels, B., \& Marks, I. (1998). A pilot study of computer-aided vicarious exposure for obsessive-compulsive disorder. Australian and New Zealand Journal of Psychiatry, 32, 268-275.

[42] Kirkby, K., Berrios, G., Daniels, B., Menzies, R., Clark, A., \& Romano, A. (2000). Process-outcome analysis in computer-aided treatment of obsessive-compulsive disorder. Comprehensive Psychiatry, 41(4), 259-265.

[43] Flavell, J. (1979). Meta-cognition and meta-cognitive monitoring: a new area of cognitive developmental inquiry. American Psychologist, 34, 906-911.

[44] Emmelkamp, P., \& Aardema, A. (1999). Metacognition, specific obsessive-compulsive beliefs and obsessive-compulsive behavior. Clinical Psychology and Psychotherapy, $6,139-145$.

[45] Purdon, C., \& Clark, D. (1999). Metacognition and obsessions. Clinical Psychology and Psychotherapy, 6, 102-110.

[46] Wells, A. (1997). Cognitive therapy of anxiety disorders: A practice manual and conceptual guide. Chichester, UK: Wiley.

[47] Wells, A. (2000). Emotional disorders and metacognition: Innovative cognitive therapy. Chichester, UK: Wiley.

[48] Wells, A., \& Mathews, G. (1994). Attention and emotion. A clinical perspective. Hove, UK: Lawrence Erlbaum \& Associates.

[49] Wells, A., \& Matthews, G. (1996). Modeling cognition in emotional disorder: The SREF Model. Behavior Research and Therapy, 34, 881-888.

[50] Fisher, P., \& Wells, A. (2008). Metacognitive therapy for obsessive-compulsive disorder: A case series. Journal of Behavior Therapy and Experimental Psychiatry, 39, 117-132.

[51] Rees, C., \& van Koesveld, K. (2008). An open trial of group metacognitive therapy for obsessive-compulsive disorder. Journal of Behavior Therapy and Experimental Psychiatry, 39, 451-458. 
[52] Moritz, S., Jelinek, L., Hauschildt, M., \& Naber, D. (2010). How to treat the untreated: effectiveness of a self-help metacognitive training program (my MCT) for obsessivecompulsive disorder. Dialogues in Clinical Neuroscience, 12, 209-220.

[53] Hayes, S., Barnes-Holmes, D., Roche, B. ., \& Eds., . (2001). Relational Frame Theory: A Post Skinnerian account of human language and cognition. New York: Kluwer Academic/Plenum.

[54] Hayes, S., Strosahl, K., \& Wilson, K. (1999). Acceptance and Commitment Therapy: An experiential approach to behavior change. New York: Guilford Press.

[55] Twohig, M. (2009). The application of acceptance and commitment therapy to the treatment of obsessive-compulsive disorder. Cognitive and Behavioral Practice, 16, $18-28$.

[56] Twohig, M., Hayes, S., \& Masuda, A. (2006). Increasing willingness to experience obsessions: Acceptance and commitment therapy as a treatment for obsessive-compulsive disorder. Behavior Therapy, 37, 3-13.

[57] Twohig, M., Hayes, S., Plumb, J., Pruitt, L., Collins, A., Hazlett-Stevens, H., \& Woidneck, M. (2010). A randomized clinical trial of acceptance and commitment therapy versus progressive relaxation training for obsessive compulsive disorder. Journal of Consulting and Clinical Psychology, 78(5), 705-716. 